\title{
High Reliability Organization in the Healthcare Industry: A Model of Performance Excellence and Innovation
}

\author{
Mountasser Kadrie ${ }^{1^{*}}$ \\ ${ }^{1}$ Program Director, Master of Healthcare Administration, Walden University
}

Received: May 9, 2017; Accepted: : May 29, 2017; Published: June 7, 2017

*Corresponding author: : Mountasser Kadrie, Program Director, Master of Healthcare Administration, Walden University. E-mail: mkadrie@gmail.com

\begin{abstract}
In recent years, the U.S. healthcare industry has been active in responding to major reform initiatives and evolving socioeconomic trends. Also, the U.S. healthcare system is vast, complex, and unique among advanced industrialized countries and at the same time the U.S. does not have a uniform health system and healthcare in the United States is provided by many distinct organizations. As a result, the healthcare delivery system is not as reliable as other industries. There is a strong business and clinical requirements to achieve a High Reliability Organization (HRO) status and to generate reliable performance results in healthcare. In this case study, the implementation and integration of an HRO model in healthcare industry in many of the sub-sets of U.S. healthcare delivery system is examined and investigated through case study approach and measurable improvements in the following outcomes: organizational effectiveness, organizational efficiency, customer satisfaction, compliance, documentation, and organizational culture are attainted. This case study affirms that HRO model facilitates innovation and promotes performance excellence and accountability in the healthcare industry.
\end{abstract}

\section{Introduction}

As a Baldrige Excellence Performance Board Examiner, the performance results of many companies from different industries including healthcare organizations who apply to the Baldrige Excellence Performance Award are assessed. The majority of these companies have implemented a High Reliability Organization (HRO) model, achieved stellar performance results, and consequently secured high reliability organization status.

The question always comes up is why the healthcare delivery system is not as reliable as other industries and especially like the airline industry? In recent years, healthcare consumers, providers, payers, and other key stakeholders have demanded better patient care and business outcomes and have asked similar questions about achieving a high reliability organization status and reliable performance results [9].

An HRO model is an environment of "collective mindfulness" where all physicians, caregivers, employees, management, and key stakeholders look for, and report, small problems or unsafe conditions before they pose a substantial risk to their patients and when they are easy and affordable to fix. Contemporary literature reveals the implementation of HRO model in clinics and physician-based practices creates improvement in the following outcomes: organizational effectiveness, organizational efficiency, customer satisfaction, compliance, documentation, and organizational culture [9]. Implementing an effective HRO model helps physician practices' leaders and practitioners design their organizations around anticipating and preventing problems [6].

As the healthcare industry sets its target to improve patient care outcomes and business performance results, it becomes clear this industry needs to invest more resources and effort to catch up to other consumer-based industries with solid track record implementing high reliability organization model. The HRO model is a mission-critical for clinics and physician-based practices to address current healthcare needs and anticipated future requirements [20]. Achieving consistent and reliable patient care outcomes and sustainable business performance results should be a shared vision and a common destiny.

In recent years, the U.S. healthcare industry has been active in responding to major reform initiatives and evolving socioeconomic trends. These initiatives and trends range from the Patient Protection and Affordable Care Act (PPACA), Accountable Care Organization (ACO), population health, bundled payment system, patient safety, the transition to valuebased reimbursement, to the rise of healthcare consumerism and increasing complexity in clinical care climate [24]. These significant forces are leading Healthcare Organizations (HCOs) including physician practices and clinics to seek better medical care coordination inside and outside their walls. These entities have learned they can benefit tremendously from embracing high reliability organization model to manage these forces and trends and addressing anticipated needs. The critical goal for clinics and physician-based practices is to ensure they are doing everything they can to improve outcomes and experiences for patients, their families, communities, and key stakeholders [27]. 
In this case study, the HRO model and its relevance to healthcare industry will be introduced. An in-depth analysis of how HRO model facilitates innovation and promotes performance excellence in the healthcare industry will be covered. Also, this paper will assess the path forward to HRO Model implementation and transformation in the healthcare industry and the risks and roadblocks that may impact how healthcare organizations use HRO model to deliver reliable, quality, and value patient care services. Finally, this paper will review the resources available to promote and implement HRO and how future trends in healthcare will shape the HRO model

\section{Discussion}

\section{HRO Definition and the Contemporary HRO Model}

What is High Reliability Organization (HRO)? Karl Weick and Kathleen Sutcliffe, who were the pioneers in HRO research, design, and implementation, provided the most compelling depiction of how high-reliability organizations can stay safe, and why they have become a model for performance, excellence, reliability, and sustainability [9]. Their definition of HRO describes an environment of

"collective mindfulness" where all employees, management, and stakeholders look for, and report, small problems or unsafe conditions before they pose a substantial risk to their organizations and when they are easy and affordable to fix. These organizations rarely, if ever, have significant accidents. They prize the identification of errors and close calls for the lessons they can extract from a careful analysis of what occurred before these events (see HRO model principles presented in Table 1).

\begin{tabular}{|l|l|}
\hline Table 1: HRO Model & \multicolumn{2}{l|}{ Explanation and Relevance } \\
\hline HRO Guiding Principles & $\begin{array}{l}\text { Embrace failures and weak } \\
\text { signals }\end{array}$ \\
\hline Reluctance to simplify & $\begin{array}{l}\text { Categories unavoidable, but } \\
\text { carried lightly }\end{array}$ \\
\hline Sensitivity to operations & $\begin{array}{l}\text { Recognize accidents are not } \\
\text { results of } 1 \text { error }\end{array}$ \\
\hline $\begin{array}{l}\text { Commitment to organization } \\
\text { resilience }\end{array}$ & $\begin{array}{l}\text { Identify, control, and recover } \\
\text { from errors }\end{array}$ \\
\hline $\begin{array}{l}\text { Deference to qualified and relevant } \\
\text { expertise }\end{array}$ & $\begin{array}{l}\text { Encourage communication from } \\
\text { all levels }\end{array}$ \\
\hline
\end{tabular}

These HRO principles point to specific weaknesses in safety protocols or procedures that can be remedied to reduce and manage the risk of future failures. Shouldn't healthcare organizations follow this model as well? Over many years, HRO has inspired hospitals, clinics, and other healthcare organizations to excel in providing safe and effective care of the highest quality. For example, many of these organizations have earned and maintained The Joint Commission's Gold Seal of Approval ${ }^{\mathrm{TM}}$, a symbol of quality recognized nationwide and reflects an organization's commitment to meeting demanding performance standards. The road to high reliability status is an ongoing journey. It is a commitment to patient safety and the way healthcare providers deliver quality healthcare [15].

\section{Comparison of Reliability and Performance Outcomes in Different Industries}

\section{Clinical-based Comparison}

To understand the value proposition of HRO and its impact on an organization's performance results in today's complex clinical and business environments, a comparison of reliability outcomes between healthcare and other industries such as the airline industry illustrates the critical need for an HRO model.

In a recent Senate hearing, a 2014 patient safety study was cited as preventable medical errors persist as the number three killer in the U.S. - third only to heart disease and cancer claiming the lives of some 400,000 people each year [2]. Do the airline and healthcare industries have similar outcomes? Despite several high-profile airline accidents, the year 2014, turned out to be a very safe year for commercial aviation, according to recently published aviation safety data. Most airline accidents in 2014 involved cargo flights (10) and passenger flights (8). Given the expected worldwide air traffic of 33,000,000 flights a year, the accident rate is one fatal passenger flight accident per 4,125,000 flights [1]. Obviously, the difference in reliability outcomes between the two industries is significant, and it should compel healthcare organizations and their leaders to consider what types of evidence-based models and strategies to use to address critical healthcare issues. Implementing a high reliability organization model is essential to address complex clinical and business challenges and opportunities [5].

\section{Business-based Comparison}

The example above focused only on the clinical outcomes of the healthcare industry, but a closer assessment of the business and operational outcomes is also warranted. Here is another aspect of the healthcare industry's unreliable business practices and weak outcomes. The Institute of Medicine in its 2011 landmark report "Better Care at Lower Cost," pointed out at least $\$ 750$ billion of the total national healthcare budget of $\$ 2.7$ trillion or 27 percent represents waste [14]. This significant waste figure is the result of poorly coordinated medical care, repeated and avoidable hospital readmissions, fragmented and costly IT infrastructure, poorly designed reimbursement models that reward volume rather than value, lack of transparency and accountability, antiquated patient flow protocols, and unreliable and outdated operations infrastructures. It is widely believed the $\$ 750$ billion is higher today than a few years ago as the total national healthcare spending in 2015 was close to $\$ 4$ trillion [25].

Recently released data from different government and private sources show an increasing number health systems, rural hospitals, clinics and physicians based practices, and academic medical centers face significant financial challenges [26]. On the contrary, the recent two years for the airline industry were the 
most profitable ever. Again, shouldn't healthcare organizations consider implementing HRO model to cut waste, improve business performance outcomes, and to enhance the financial position and viability?

\section{Healthcare Industry in a Dynamic Transformation}

The U.S Economy and Healthcare Industry are in Transition. In the last decade, the U.S. economy and society have been in a historic transformation phase and undergoing significant structural changes. New technologies, the rise of globalization, aging population and baby boomers, and the increase of consumerism are examples of these major structural changes. The healthcare industry also is no exception, and it has been going through a constant transition and dynamic change. When facing the challenge and vast opportunity, healthcare organizations not only must contend with new demanding incremental demographic shifts that profoundly modify and impact the population's health status, but also with rising healthcare consumerism and expectations [19]. The healthcare industry never in its history has it seen such a significant disruption of its clinical and business processes by embracing new technologies and clinical advances in its day-to-day operations.

As the U.S. economy transitions itself due to emerging business, population, socioeconomic and political trends and forces, many industries have successfully developed sustainable, reliable, and systematic strategic and operational plans to cope with these evolving trends and forces. The airline industry after the $9 / 11$ attacks in 2001 and the great economic recession of 2009 has successfully been able to survive and thrive. This industry successfully managed to address complex operational, logistical, security, and resources demands despite the fact of facing challenging business conditions, complex and costly government mandates and regulations, and global competitive forces. At the same time, no one can ignore the fact the healthcare industry has made noticeable strides and improvements over the last decade. However, the results achieved do not quite simply justify or support the investment, resources, and or efforts made. In other words, the healthcare industry is not where it should be when it comes to being a high reliability industry [29].

\section{Transformation Implications}

Currently, the healthcare industry including physician practices and integrated health systems are going through dynamic transition moving from volume to value-based reimbursement system and promoting population healthcare delivery model [28]. In clinics and physician practices, the overarching goal for providers must be improving the value proposition for patients, where the value is defined as the health outcomes achieved matter to patients relative to the cost of reaching those clinical outcomes [27].

Under this transition, the current White House administration is embracing a vision for the future of the healthcare industry and in particular the Medicare program by committing to clear goals and a timeline for shifting Medicare payments from volumeto-value. It is predicted the new administration in the White
House after the 2016 election will continue this commitment. In this environment, medical providers need a reliable strategy to transcend traditional cost reduction approaches and responds to new payment models. If providers can improve and sustain patient outcomes, they can sustain or grow their market share. If they can improve the efficiency and reliability of providing excellent medical care, they will enter any contracting discussion from a position of competitive strength [19]. Those providers who improve value will be the most competitive. Clinics, physicianbased practices, and integrated health systems that fail to enhance value proposition, no matter how prestigious and influential they seem today, are likely to encounter growing competitive pressure and challenges [24].

To leverage this impactful change and to manage its requirements, healthcare providers should embrace HRO model [11]. It is believed healthcare organizations and their leaders need to do more to change the status quo and to become high reliable organizations and to learn best practices from other industries that have achieved consistent reliability and performance results. The airline industry has worked tirelessly on these problems (delays, over-processing, redundant work, poor inventory management, inefficient transport, cost overruns, overproduction, and defects that cause harm and re-work) for decades. Although its operations and flow patterns are significantly less complex than healthcare, it has mastered basic elements where healthcare industry can learn from to give it a jump-start on mastering and managing a difficult but necessary components of operational design that will lead to reliable and improved outcomes at lower costs. i.e., value-based proposition [25].

\section{Analysis}

\section{Embracing HRO Model Facilitates Innovation in the Healthcare Industry}

Innovation is an essential component of high reliability organization's DNA, and it is critical to sustaining integrated health systems as healthcare moves from volume to value [4]. Integrated healthcare delivery systems see their implementation of HRO model as a conduit to promote innovation as an essential component of their corporate strategy and long-term survival. These systems have leveraged their clinical and technological expertise and know-how to develop innovative healthcare services and implement best clinical and business practices and programs [3].

The case calling for the healthcare industry to embrace HRO model and innovation is starting to pay off in meaningful and incremental successes. These successes in recent years affirm the establishment of the relationships between Weick and Sutcliffe's HRO principles and desired clinical and business behaviors of healthcare providers. Some of these practices and examples about the HRO principles are presented below in (Table 2). 


\begin{tabular}{|c|c|c|}
\hline $\begin{array}{l}\text { HRO Guiding } \\
\text { Principles }\end{array}$ & $\begin{array}{l}\text { Corresponding } \\
\text { Behavior of } \\
\text { Care Providers }\end{array}$ & $\begin{array}{l}\text { Examples in Healthcare } \\
\text { Industry }\end{array}$ \\
\hline $\begin{array}{l}\text { Preoccupations } \\
\text { with failure }\end{array}$ & Attitude & $\begin{array}{l}\text { RNs and other clinical care } \\
\text { professional and providers ask } \\
\text { if physicians have marked the } \\
\text { correct surgical site. }\end{array}$ \\
\hline $\begin{array}{l}\text { Reluctance to } \\
\text { simplify }\end{array}$ & $\begin{array}{l}\text { Metacognitive } \\
\text { skills }\end{array}$ & $\begin{array}{c}\text { Attending residents and fellows } \\
\text { discuss roles, responsibilities, } \\
\text { and expectations for the when } \\
\text { a } \\
\text { critical patient is admitted. }\end{array}$ \\
\hline $\begin{array}{l}\text { Sensitivity to } \\
\text { operations }\end{array}$ & $\begin{array}{l}\text { Systems- } \\
\text { based value } \\
\text { proposition and } \\
\text { practices }\end{array}$ & $\begin{array}{l}\text { Outgoing RN shift teams } \\
\text { inform the incoming RN teams } \\
\text { of patients' status, both } \\
\text { incoming and out coming } \\
\text { teams summarize info and } \\
\text { confirm accuracy for better } \\
\text { patient clinical outcomes. }\end{array}$ \\
\hline $\begin{array}{l}\text { Commitment } \\
\text { to organization } \\
\text { resilience }\end{array}$ & $\begin{array}{l}\text { Leadership and } \\
\text { team focus on } \\
\text { competency } \\
\text { skills }\end{array}$ & $\begin{array}{l}\text { Nurse team leader monitors a } \\
\text { physician's performance } \\
\text { during a procedure to ensure } \\
\text { protocol steps are not omitted } \\
\text { when delivering patient care } \\
\text { services. }\end{array}$ \\
\hline $\begin{array}{l}\text { Deference } \\
\text { to qualified } \\
\text { and relevant } \\
\text { expertise }\end{array}$ & $\begin{array}{l}\text { Emotional } \\
\text { intelligence; } \\
\text { advocacy, } \\
\text { accountability, } \\
\text { and assertion }\end{array}$ & $\begin{array}{l}\text { Nurses more than ever before } \\
\text { are empowered to question a } \\
\text { physician's medication order } \\
\text { because of a patient's known } \\
\text { Allergies or adverse reaction. }\end{array}$ \\
\hline
\end{tabular}

Intermountain Healthcare is a non-profit healthcare system and is the largest healthcare provider in the Intermountain West, used HRO techniques to redefine value. They used "Hotspotting"-identifying high-risk patients through medical data and helping them to stay out of the hospital setting. So did medical home primary care, multispecialty clinics for patients and integrating behavior health into primary care. Helping patients to manage diabetes and high blood pressures better by integrating pharmacy, nutrition and lifestyle coaching into primary care visits, generated some of the biggest savings for Intermountain.

By focusing on bedside shift reports and improving communication with patients, providers, among other things, nurses at seven Washington state hospitals and clinics were able to make a significant impact in the work they do. The American Association of Critical-Care Nurses recently reported the result from its Clinical Scene Investigator Academy, a 16-month, team-based program to encourage RNs as leaders and change champions in their clinics. Among the success, those involved in the program were able to decrease medication errors, eliminate patient's falls, and increase patient mobility, with a projected cost saving of almost $\$ 570,0000$.

For HRO model to deliver the desired performance results healthcare systems and clinics must have in place capable and robust IT structures to facilitate clinical data exchanges between providers and other stakeholders [9]. These days the diversity of information systems in healthcare delivery setting presents challenges to patient data sharing. Interoperability among disparate vendor applications remains one of the most significant roadblocks to providing continuity of care across the clinical setting [28]. These roadblocks are driven by many complex issues including the lack of clinical IT standards, high integration costs for physician practices and other non-acute services, and lack of electronic health record automaton in nursing homes and urgent care centers. The American Hospital Association (AHA) Report: "Why Interoperability matters, 2015," indicates increasing number of integrated health systems starting to set up standards to share information for care coordination with nonaffiliated ambulatory providers. Many of these health systems are HRO adopters and in their pursuit of information sharing, they have implemented systematic protocols and standards to facilitate clinical data exchange.

UPMC, the well-known Pittsburgh-based healthcare network, is committed to HRO model as it leverages its investments to improve patient outcomes, increase efficiency, reliability, and quality, and to reduce healthcare costs. The commitment to HRO model is a mission-critical innovation and it is expected to shape behaviors for the rising risk population. UPMC is expected to go live soon with a substantial enhancement to its telehealth program. UPMC will deploy a comprehensive cloud-based platform to facilitate virtual care visits to those with chronic diseases.

The Advocate Healthcare, faith-based, the not-for-profit health system based in Downers Grove, Illinois, has operated as a value-based medical care organization for several years. This integrated system is moving from fee-for-service revenue models to value-based reimbursement and leading Advocate toward adopting sophisticated clinical data exchanges and analytics systems to quantify quality measures and track process improvement. The Advocate had a big problem with siloed and fragmented data spread across many EHR systems that did not integrate well together. With the goal of becoming a high reliable organization, Advocate was motivated to improve patient care and control costs and decided to invest in partnership with a national healthcare information technology vendor and set up a cloud-based analytics platform to integrate data from all the EHRs within Advocate's existing information technology infrastructure.

\section{Embracing HRO Promotes Performance Excellence in the Healthcare Industry}

For most healthcare systems, reducing patients' readmissions and tracking care transitions are central focus targets as these systems work diligently to improve clinical outcomes, enhance the patient experience, and to reduce overall costs [30]. High reliability organizations such as the Hartford HealthCare, a large tertiary-care teaching hospital system in Hartford, Connecticut, is investing significant resources and effort addressing reducing readmissions and transition in care. In healthcare no two patients are identical, and it is important to consider and treat every patient's unique health needs, risks, social determinates of health, 
community and other factors [17]. Knowing a particular patient to focus on and how is a true challenge. Healthcare delivery system is also fragmented, so being able to share information as patients transition between medical care settings is paramount [13]. To help improve care transition and, ultimately, reduce readmissions, the Hartford HealthCare implemented advanced clinical decision support software system to track results.

Leaders of Hartford HealthCare system formed a crossfunctional team. These teams included nurses, physicians, case managers, social workers, clinical trainers, business executives and informational technologists from across the organization. The team main task was to ensure the new clinical decision support system tracks and evaluates existing clinical and patient care workflows. Adopting this strategy have allowed Hartford HealthCare to reap many strategic benefits ranging from improved discharge planning achieving and improving population health management outcomes. This high reliability organization component implemented at this system has served as the catalyst for a cultural shift that resulted in an improved, streamlined care transition process and less variation across the system. These benefits will be the underpinning of high reliability organization's success in managing population health needs, improving outcomes, and controlling overall healthcare costs [2].

HRO model adoption not only creates an opportunity for innovation in technology, data analytics, and IT infrastructure but also promotes innovation opportunities in many other areas of the healthcare delivery ecosystem [25]. In recent years, increasing number of outpatient facilities has embraced Lean design to improve patient throughput. Porter and Lee delineate nowadays in an ambulatory care setting, what it was done in the outpatient arena is what it used to be done 10 years ago in an inpatient setting [24]. As ambulatory care has flourished, the U.S. healthcare market has focused increasingly on efficiency and the patient experience. Health systems implementing HRO model are building ambulatory care facilities throughout their communities, rather than just on the main hospital campus-as patient convenience and a healthcare organization's visibility in the community are now key factors in choosing preferred ambulatory care sites [29]. Off-site ambulatory facilities also can make health and wellness services more accessible to patients, improve the reliability of patient care delivery, and increase patient satisfaction. Children's Hospital of Philadelphia has built ambulatory clinics off the main campus to improve the patient care experience and takes away patients' anxiety of going to the main hospital campus.

While many healthcare systems have pursued a strategy of providing community-based care in a more accessible, convenient and less intimidating environment, implementing HRO model has also provided health systems, clinics, and physician practices the opportunity to focus on facility design and patient care experience [7]. These organizations conduct extensive research to listen to patients and customers and understand how they interact with and perceive healthcare spaces, how healthcare providers apply treatments, therapies, and procedures, and how to standardize clinical spaces within buildings and across their institutions in different site locations and settings. The reason is, these systems are now having their staff travel from location to location. Therefore, a standardized clinic module can support a variety of specialties and is easy for people who work at multiple sites to navigate [17]. Many clinic rooms are now designed for efficient and comfortable care. They are built both for exams and consultations, so patients and physicians do not have to move to another room for a consult.

As payment shifts to value-based models, increasing number of healthcare organizations will pursue lower-cost settings more aggressively than before while employing creative approaches to distributing and delivering medical care [22]. Every healthcare system attempting to start a new medical facility should utilize Lean methodology [7]. Not only does the process help organizations design efficient operational workflows and the necessary auxiliary facilities to support them, but it also stresses continuous improvement and support of HRO model [27]. The St. Louis Children's Specialty Care Center is one of the only of few stand-alone, comprehensive outpatient facilities in U.S. focused exclusively on patients up to the age of 21 and it is 12 miles from the main St. Louis Children's Hospital campus in a suburban area. This center is an example of how HRO model can fosters innovation to provide comprehensive community-based care in a more accessible, convenient, and more welcoming environment.

The HRO model implementation impact is reaching beyond the basic clinical care setting to medical insurance payers integrated across the entire continuum of medical care. The Accountable Care Organization transformation in recent year has given these insurance payers the opportunity to work directly and collaboratively with hospitals and physician practices in each community. This collaborative effort critical in supporting practice transformation from a reactive model of patient care to a proactively accountable care, analyzing population data to measure, monitor, and manage the healthcare of patients and present results and outcomes to these organizations [9]. United Healthcare Accountable Care Practice consultants and teams work collaboratively and directly with various healthcare systems and physician practices to improve access to medical care. They use practice level population data to analyze overall capacity/demand for appointment and visits patterns by practice clinic site and assist the practice leadership to implement process improvement to reach mutual goals and improve patient access. These teams help hospitals reduce inappropriate ER use by using the hospital's Admission, Discharge, and Transfer (ADT) and daily ER data to analyze trends and outliers and work with all community partners to implement proactive strategies to address issues, and measure impact using Rapid Cycle Improvement, PDCA (Plan-Do-Check-Act) approach.

These teams engage and coordinate with hospitals' clinical care teams to reduce avoidable admissions and readmissions by tracking and managing medical care transitions of all discharges and ensure follow-up with Primary Care Physicians (PCPs) and or specialists within seven days. These teams use predictive modeling analysis of practice population to improve the medical care of high-risk patients and assist practices in identifying 
cohorts of high-risk health home patients for focused and enhanced improvement initiatives.

Also, United Healthcare clinical teams and consultants support various healthcare systems and practices in implementing process improvements to assist the practice in becoming a certified Patient Centered Medical Home (PCMH). This is another valuable HRO approach leveraged where United Healthcare engages with many health systems by assisting them in creating workflows to optimize care delivery, introducing best practices improvements, and evaluating outcomes using rapid cycle improvement PDCA method.

\section{Assessment}

Health systems, physician practices, and clinics in the United States are facing now and going forward an unparalleled force to change, to become safer and more efficient [8]. Creating a safe and high reliability environment requires healthcare leaders recognize safety should be the root cause of achieving efficiency, reliability, and effectiveness. If the inverse of this relationship exists, the likelihood of creating unsafe yet highly efficient processes increases. Only if safety is the starting point can the correlations among safety, efficiency, and effectiveness remains positive [30].

\section{The Path Forward to HRO Model Implementation and Transformation}

A healthcare organization whether it is an integrated health system, clinic, or physician practice is a complex environment setting that brings together a wide range of people with diverse needs, skills, and expectations [24]. Many clinics and physician practices are microcosms of their communities, and this leaves a significant opportunity for risk as well as for making sure medical practice issues go right and do not cause patient care adverse outcomes. Clearly, this is why HRO model matters because patient's safety initiatives are everybody's business, and it is the cornerstone of the HRO model in the healthcare industry [31].

The National Patient Safety Foundation's recent report, "Free from Harm: Accelerating Patient Safety Improvement Fifteen Years after "To Err Is Human," proposed greater oversight, communication, funding, and support to help improve patient safety and the reliability of patient care delivery models. Integrated health systems and clinics can make substantial progress toward high reliability organization model by undertaking several specific organizational change initiatives. The leaders of these organizations must continually challenge and improve upon themselves and how they respond to reliability and performance concerns. This improvement will ultimately drive a shared sense of resilience throughout the organization [27].

\section{Managing Risks and Roadblocks}

Implementing HRO model comes with a set of common roadblocks and challenges identified by all the organizations pursuing high reliability [20]. Healthcare organizations and their leaders must develop robust and sustainable capabilities to manage these challenges as many of these characteristics provided below need to exist in the average healthcare setting.

\section{Hypercomplexity}

HROs exist in complex and demanding environments, and they depend on using qualified multi-team clinical and business systems to coordinate for safety and patient and employees wellbeing. The safety of a hospitalized patient depends primarily on the effective coordination of care providers such as physicians, nurses, pharmacists, medical technicians, technicians who maintain equipment, support staff who provide meals and maintain the physical environment, and others [26].

\section{Tight coupling}

HROs consist of tightly coupled teams. These teams depend primarily on tasks performed across their team [2]. For example, a safe surgery in an acute facility or day-surgery center depends on the ability of nurses, technicians, the surgery team, housekeeping, and transport to coordinate their efforts so the patient arrives in surgery at the right time, with the proper preparation, and with the right supplies available for the operation to proceed smoothly. Every healthcare leader recognizes this coordination is critical but is often far from perfect.

\section{Extreme hierarchical differentiation}

In HROs, roles are clearly differentiated and defined. Intensive coordination efforts are needed to keep members of the teams working cohesively and continuously. During times of crisis, however, decision making is deferred to the most knowledgeable staff in the medical care or business team, regardless of their position in the organization [9].

\section{Multiple decision makers in a complex communication network}

HROs consist of many decision makers working to make significant, interconnected decisions. Like all clinics and hospitals, HROs must develop systematic processes to allow these decision makers to communicate effectively with each other [11].

\section{High degree of accountability}

HROs have a high level of accountability when an error occurs it has severe consequences [2]. In this respect, health systems and clinics differ somewhat from many traditional HROs, because medical errors and adverse clinical events tend to affect a single patient or group of patients rather than large groups of people at once. Moreover, despite flawless care, patients in medical setting do die, so distinguishing those whose deaths were inevitable from those whose deaths the hospital or clinic could have averted is not easy [28].

\section{Need for frequent and immediate feedback}

HROs exist in industries where team members must receive frequent and regular feedback at all times. This feedback and the opportunity to make continuous adjustments and improvement based on it are essential to anticipate and avert potential problems and adverse events before they become crises.

Health systems and clinics employee large numbers of staff 
and utilize expensive physical resources. Providing this type of feedback to staff and for them to function as an HRO, they need responsive clinical and business systems in place and a mindset to allow multiple stakeholders to receive and respond to relevant feedback, rather than being overwhelmed by information [13].

\section{Compressed time constraints}

Time constraints are common to many industries, including healthcare. In HROs, the systems and culture allow staff and teams to identify when they lack time to complete all needed tasks reliably and obtain additional assistance [5]. Hospitals and clinics face the same challenge but do not always have staff with the appropriate resources and training needed to maintain high reliability status when confronted with a significant time constraint.

\section{Implementing HRO is a Mission Critical}

The implementation of HRO model must be mission-critical for the healthcare organization, and it must be led by the CEO, who is the primary stakeholder one who can drive the necessary change mandate across an organization [10]. The CEO needs help in getting an outside-in view: the riskiest situation is to think HRO transformation in the vacuum of an environment that healthcare organizations have already known. It should be acknowledged the path to implementing HRO is challenging for many healthcare organizations. Many of these organizations face daunting roadblocks. However, let's imagine what the costs will be if HRO model is not implemented.

\section{Change Management Drives Successful HRO Implementation}

The biggest mistake is underestimating the magnitude of the change required to implement HRO model. Culture change must start at the top because while there are now in the organization successful executives and leaders thriving at managing in a certain way, this way may not make the organization successful in the future [20]. The implementation of an HRO model begins with defining a strategy, but it does not end there. HRO implementation extends from strategy development through execution-in the process to realize sustainable competitive advantage and to generate measurable results [10].

The Kotter's 8-Step Process for Leading Change presented in (Table 3) serves an effective conduit for leaders and organizations trying to transform or execute their strategies [18]. Both the initial Leading Change 8-Step Process (1996) and Accelerate 8-Step Process (2014) models can serve the needs of the healthcare industry to facilitate and implement HRO model. By applying the Kotter's 8-step

Process, healthcare leaders and organizations should gain unprecedented decision-making authority and leverage, the risks of doing so, and the rewards of an exponentially more efficient and more resilient organization.

\begin{tabular}{|c|c|}
\hline $\begin{array}{l}\text { Leading Changes 8-Step } \\
\text { Process (1996) }\end{array}$ & Accelerate 8-Step Process (2014) \\
\hline $\begin{array}{l}\text { Respond to or affect episodic } \\
\text { change in } \\
\text { rigid, finite, and sequential } \\
\text { ways }\end{array}$ & $\begin{array}{l}\text { Run the steps concurrently and } \\
\text { continuously }\end{array}$ \\
\hline $\begin{array}{l}\text { Drive change with a small, } \\
\text { powerful core group }\end{array}$ & $\begin{array}{l}\text { Form a large volunteer army from } \\
\text { up, down } \\
\text { and across the organization to serve } \\
\text { as the } \\
\text { change engine }\end{array}$ \\
\hline $\begin{array}{l}\text { Function within a traditional } \\
\text { hierarchy }\end{array}$ & $\begin{array}{l}\text { Function in a network flexibly and } \\
\text { agilely } \\
\text { outside, but in conjunction with, a } \\
\text { traditional hierarchy }\end{array}$ \\
\hline $\begin{array}{l}\text { Focus on doing one new thing } \\
\text { very well in a linear fashion } \\
\text { over time }\end{array}$ & $\begin{array}{l}\text { Constantly seek opportunities, } \\
\text { identify } \\
\text { initiatives to capitalize on them, and } \\
\text { complete them quickly }\end{array}$ \\
\hline
\end{tabular}

\section{The Future of HRO}

Many healthcare industry experts have projected multiple and intersecting pressures will drive the transformation of the healthcare delivery system to focus on quality, reliability, and safety and transitioning from volume to value-based payments over the next decade [28]. In the current regulatory and business environments, health systems and clinics must focus their efforts and limited resources on promoting performance initiatives that are essential in the short term, and this will also remain critical for long-term success. In recent years, an increasing number of health systems and large size clinics are investing additional resources to pursue must-do strategies to succeed in the Future [25]. The strategy of pursuing HRO is a critical one to support the core organizational competencies many healthcare organizations should establish to remain successful and competitive in this time of sweeping change [21].

\section{Predictive Medicine and Big Data}

In recent years, the increasing role of Health Information Technology (HIT), big data, and predictive analytics in healthcare has been instrumental in implementing HRO model [9]. HIT has allowed health systems and their integrated clinics to leverage clinical data to more precisely monitor their systems of care, the dispensing of medications to patients, and the amount of system waste [28]. These data have focused attention on the frequency with an ideal care is not provided to patients. HIT has also impacted clinics in another way. Clinics embracing HIT systems have found automating flawed systems can make their operations less efficient rather than more. Therefore, making HIT systems reliable before they are automated has become a priority [12].

Integrated health systems can leverage data analytics to make significant improvements in clinical and business outcomes [25]. Recent findings have indicated the rapid growth of predictive analytics enables health systems and clinics to spot trends from 
avoidable patient readmissions to staffing and physical resources needs. Predictive analytics enabled the Robert Wood Johnson University Hospital (RWJUH) and its many clinics in 2016 to save $\$ 10$ million using workforce analytics by addressing staff issues and increasing employee engagement. Also, RWJUH has also increased employee engagement and reduced staffing issues without layoffs. This health system needed and used clinical and business data to help in many areas such as improving operations, making evidence-based decisions and taking the most appropriate action, and eventually implementing an effective HRO model.

\section{Valuable Resources for HRO Implementation}

Health systems and clinics can leverage many available resources to initiate and implement HRO. The Joint Commission's (www.jointcommission.org) knowledge of healthcare organizations with practitioner knowledge gained from the industry literature, experts in high-reliability industries, and leading safety scholars outside healthcare industry offers healthcare organizations evidence-based foundation to develop a conceptual and practical framework for assessing their readiness for and progress toward high reliability [16].

Also of an added value for healthcare organizations attempting to implement HRO is to consider the Baldrige Criteria for Performance Excellence, Core Values and Concepts, and Guidelines for evaluating an organization's processes and results (www.nist.gov/baldrige/). The recent updated Baldrige Criteria Program features a renewed focus on managing all of the components of the organization as a unified whole; managing change; and dealing with data analytics, data integrity, and cyber security. The ratio of the Baldrige Program's benefits for the U.S. economy to its costs is estimated at 820 to 1 . Many healthcare organizations that employ the Baldrige Criteria outperform their non-Baldrige peer systems by achieving faster 5-year performance improvement than peers. Whether used as guidance in establishing an integrated performance management system or for self-assessing progress or benchmark purposes the Baldrige Criteria Program is about helping healthcare organizations, no matter what size or type, innovate, improve, and become Reliable [23].

\section{Conclusion}

In conclusion, there is a well established strategic need or healthcare providers (hospitals, physician practices, and clinics) to make evidence-based changes to increase successful operations and functions to become recognized as high reliability organizations. In the U.S. healthcare industry there many Integrated Health Systems (IHS) that operate complex vertical and horizontal sub-systems such as hospitals, physician practices, ambulatory care, specialty hospitals and surgical centers, nursing homes and assisted living facilities. These IHSs need to leverage its pioneering and innovative work in clinical process improvement and advanced information system technology, data analytics, and operation infrastructure capabilities to implement HRO model and to transform itself.
In this case study, we learned that implementing evidencebased HRO model has many strategic and tactical benefits and it could serve as a successful model for healthcare systems transformation and innovation across the country. The roads to success in HROs depend on dedicated and relentless leadership that puts quality, safety, and excellence as the central focus of strategic success. Health systems like Intermountain, UPMC, Cleveland Clinic, RWJUH, and others have revamped clinical and business operations throughout the care delivery ecosystem. Empirical research and lessons learned from the practitioner setting have shown HRO model is beginning to provide insight into the context of medical care to influence reliability [29]. This insight has been instrumental for healthcare organizations to tie organizational results back to their purpose and worthwhile work; these organizations more than ever before are inspired to achieve great results and become highly reliable.

\section{References}

1. Federal Aviation Administration. 2016.

2. Agency for Healthcare Research and Quality. 2016.

3. A new model for ANCC's Magnet recognition program. ANCC. 2016.

4. Brown B, Crapo J. The Key to Transitioning from Fee-for-Service to Value-Based Reimbursement. 2016.

5. Burton DA. Driving out waste: a framework to enhance value in clinical care. Healthc Financ Manage. 2013;67(6):94-99.

6. Colla $\mathrm{CH}$. Swimming against the current--what might work to reduce low-value care? N Engl J Med. 2014;371(14):1280-1283. doi: 10.1056/ NEJMp1404503

7. Eagle A. Designing Lean ambulatory care facilities. 2016 .

8. Ellison A. Moody's: 3 challenges facing the healthcare industry in 2016. 2016.

9. Griffith JR. Understanding High-Reliability Organizations: Are Baldrige Recipients Models? J Healthc Manag. 2015;60(1):44-61.

10.Geffner JC. Reinventing Healthcare: 5 Strategies for Successfully Leading Change. 2014.

11. Guterman S. Leaving fee-for-service behind: Moving toward pay-forperformance. 2013.

12. Hospital Consumer Assessment of Healthcare Providers and Systems. 2015.

13. Institute for Healthcare Improvement. 2016.

14. Institute of Medicine. 2016.

15. Institute for Safe Medication Practices. 2016.

16. The Joint Commission. 2016.

17. Joshi MS, Ransom ER, Nash DB, Ransom SB. The healthcare quality book: Vision, strategy, and tools. 3rd ed. Chicago, IL: Health Administration Press;2014.

18. Kotter. The 8-Step Process for Leading Change.2016. 
19. May EL. The power of zero: steps toward high reliability healthcare. Healthc Exec. 2013;28(2):16-18, 20, 22.

20. McLees AW, Nawaz S, Thomas C, Young A. Defining and assessing quality improvement outcomes: a framework for public health. Am J Public Health. 2015;105 Suppl 2:S167-73. doi: 10.2105/ AJPH.2014.302533

21. Merrild P. The Biggest U.S. Health Care Challenges Are Management Challenges. 2015

22. Moore KD, Eyestone K, Coddington DC. The healthcare cost curve can be bent. Healthc Financ Manage. 2013;67(3):78-84.

23. NIST. Baldridge: Improve your performance: The 28th annual Quest for Excellence conference. 2015.

24. Porter ME, Lee TH. The strategy that will fix health care. Harvard Business Review. 2013.

25. PwC's Global Data and Analytics Survey 2016. PWC. 2016.

26. Sabbatini AK, Nallamothu BK, Kocher KE. Reducing variation in hospital admissions from the emergency department for lowmortality conditions may produce savings. Health Aff (Millwood). 2014;33(9):1655-1663. doi: 10.1377/hlthaff.2013.1318
27. Swensen S, Pugh M, McMullan C, Kabcenell A. High-impact leadership: Improve care, improve the health of populations, and reduce costs. IHI White Paper. Cambridge, Massachusetts: Institute for Healthcare Improvement. 2013.

28. The Leapfrog Group. 2016.

29. Trbovich P. Five ways to incorporate systems thinking into healthcare organizations. Biomed Instrum Technol. 2014;Suppl:31-34, 36. doi: 10.2345/0899-8205-48.s2.31

30. Tsai TC, Joynt KE, Orav EJ, Gawande AA, Jha AK. Variation in surgicalreadmission rates and quality of hospital care. $N$ Engl J Med. 2013;369(12):1134-1142. doi: 10.1056/NEJMsa1303118

31. U. S. Department of Health and Human Services. Hospital Compare: A quality tool provided by Medicare. 2016.

32. Eric c. Ultimate Guide to Medicare. 